\title{
The v-ErbA oncoprotein quenches the activity of an erythroid-specific enhancer
}

\author{
Georgia G Braliou ${ }^{1}$, Paolo Ciana ${ }^{1,3}$, Willem Klaassen ${ }^{1}$, Olivier Gandrillon ${ }^{2}$ and \\ Hendrik G Stunnenberg*,1 \\ ${ }^{1}$ Department of Molecular Biology, NCMLS University of Nijmegen, Geert Groote plein 26 PO Box 9101, 6500 HB Nijmegen, The \\ Netherlands; ${ }^{2}$ Centre de génétique moléculaire et cellulaire, UMR CNRS 5534, Université Claude Bernard Lyon I, Bat 741, 43, \\ boulevard du 11 Novembre 1918, 69622 Villeurbanne Cedex France
}

v-ErbA is a mutated variant of thyroid hormone receptor (TR $\alpha /$ NR1A1) borne by the Avian Erythroblastosis virus causing erythroleukemia. $T R \alpha$ is known to activate transcription of specific genes in the presence of its cognate ligand, T3 hormone, while in its absence it represses it. vErbA is unable to bind ligand, and hence is thought to contribute to leukemogenesis by actively repressing erythroid-specific genes such as the carbonic anhydrase II gene (CA II). In the prevailing model, v-ErbA occludes liganded TR from binding to its cognate elements and constitutively interacts with the corepressors NCoR/ SMRT. We previously identified a v-ErbA responsive element (VRE) within a DNase I hypersensitive region (HS2) located in the second intron of the CA II gene. We now show that HS2 fulfils all the requirements for a genuine enhancer that functions independent of its orientation and position with a profound erythroid-specific activity in normal erythroid progenitors (T2ECs) and in leukemic erythroid cell lines. We find that the HS2 enhancer activity is governed by two adjacent GATAfactor binding sites. v-ErbA as well as unliganded TR prevent HS2 activity by nullifying the positive function of factors bound to GATA-sites. However, v-ErbA, in contrast to $T R$, does not convey active repression to silence the transcriptional activity intrinsic to a heterologous tk promoter. We propose that depending on the sequence and context of the binding site, v-ErbA contributes to leukomogenesis by occluding liganded TR as well as unliganded TR thereby preventing activation or repression, respectively. Oncogene (2001) 20, 775-787.

Keywords: v-ErbA; thyroid hormone; repression; erythroid enhancer; carbonic anhydrase II; GATA-sites

\section{Introduction}

Malignant transformation of hematopoietic cells is caused by the disturbance of a delicate balance between

\footnotetext{
*Correspondence: H Stunnenberg

${ }^{3}$ Current address: Institute of Pharmacological Sciences, University of Milan, Via Balzaretti 9, 20133 Milan, Italy

Received 22 June 2000; revised 22 November 2000; accepted 6 December 2000
}

proliferation and differentiation. The correct functioning of members of the nuclear hormone receptor superfamily is required to maintain homeostasis, and to promote differentiation of hematopoietic cells. Not surprisingly, mutated, aberrant nuclear receptors are found in many types of cancer and diseases (Wolff, 1997; Goldhirsch and Gelber, 1996). Paradigms are fusion proteins involving retinoic acid receptor $(\mathrm{RAR} \alpha), \mathrm{PML}-\mathrm{RAR} \alpha, \mathrm{PLZF}-\mathrm{RAR} \alpha$ and NPM-RAR $\alpha$ that cause human acute promyelocytic leukemia (APL) (review Warrell et al., 1993). v-ErbA is an oncogenic version of chicken thyroid hormone receptor $(\mathrm{TR} \alpha)$ and is one of the two co-operating oncogenes of the Avian erythroblastosis virus (AEV) which causes fatal avian erythroleukemia (AEL) in chickens (for review Beug et al., 1996; Stunnenberg et al., 1999). The second oncogene of AEV is $\mathrm{v}$-ErbB, which is a mutated epidermal growth factor receptor (EGFR) with constitutive tyrosine kinase activity. While v-ErbB alone causes a partial differentiation arrest and a delayed, weak erythroleukemia, v-ErbA by itself transforms erythroid progenitor in vitro (Gandrillon et al., 1989) but induces disease in vivo only when sufficiently overexpressed (Casini and Graf, 1995; for review Beug et al., 1996). The transformation promoting activity of $\mathrm{v}$-ErbA is widely thought to lie in its ability to arrest differentiation of $\mathrm{v}$-ErbB-expressing erythroblasts by effectively silencing stage-specific erythroid genes such as the carbonic anhydrase II gene (CA II) that are otherwise activated by liganded TR (Pain et al., 1990; Disela et al., 1991; Zenke et al., 1990).

$\mathrm{TR} \alpha$ (NR1A1) (Nomenclature committee, 1999), acts as a ligand-operated molecular switch; firstly, in the absence of ligand, TR is proposed to repress transcription via association with corepressors such as NCoR/ SMRT (Horlein et al., 1995; Chen and Evans, 1995). The corepressors, in turn, recruit histone deacetylase (HDAC)-containing complex(es) that stabilise(s) repressive, 'closed' chromatin structure to assure efficient silencing of downstream genes (Heinzel et al., 1997; Wong et al., 1998). Second, upon ligand binding, the receptor undergoes a conformational change that causes dissociation of the corepressor complex(es) and permits subsequent association of coactivator complexes such as p300/CBP, SRC and TRAP/SMCC to activate transcription (Ogryzko et al., 1996; Bannister 
and Kouzarides 1996; for review Glass and Rosenfeld, 2000). Hence, the ability of v-ErbA to transform erythroid progenitors can be attributed to its unliganded 'mode' displaying a constitutive repressive activity by antagonising T3-mediated transcriptional activation (Damm et al., 1989; Sap et al., 1989; Horlein et al., 1995; Chen and Evans, 1995; for review Stunnenberg et al., 1999; Thormeyer and Baniahmad, 1999). The fact that many of the mutations in v-ErbA abolish activation functions present in genuine $\mathrm{TR} \alpha$ (NR1A1) (Barettino et al., 1994) is consistent with the occlusion/corepressor model. Although attractive, this model awaits in vivo verification; thus far, supporting data have been obtained almost exclusively from transient transfection assays, two-hybrid assays and in vitro GST-pull down experiments. One observation in particular is difficult to reconcile with this model. vErbA, as compared to TR, shows lower affinity and altered DNA specificity for classical thyroid responsive elements (TREs) mainly due to a mutation in the DNA-recognition helix (P-box) (Judelson and Privalsky, 1996; Subauste and Koenig, 1995; Wahlstrom et al., 1996). Reversion of this mutation restores wild type (TR) DNA binding affinity. However, rather than acting as a superoncogene (due to increased DNA binding properties), the P-box-reverted v-ErbA variant has lost the ability to transform erythroid cells (Nelson et al., 1994; Sharif and Privalsky, 1991; Bauer et al., 1997). These observations imply that v-ErbA does not merely function as a constitutive unliganded TR; moreover, the set for $\mathrm{v}$-ErbA binding sites appears to be distinct from or only partially overlapping with that of TR.

In a previous study, we identified a DNase I hypersensitive site (HS2) in the second intron of the chicken carbonic anhydrase II (CA II) gene (Ciana et al., 1998). CA II is a direct target gene of v-ErbA and deregulation of CA II transcription partially accounts for the v-ErbA induced phenotype (Fuerstenberg et al., 1992). Our data suggested that HS2 might act as an enhancer. We showed that the v-ErbA oncoprotein bound to a v-ErbA responsive element (VRE), located within the HS2, prevented the activity of the enhancer. Here, we report on a detailed analysis of the CA IIHS2 enhancer that provides insight into the mechanism by which $\mathrm{v}$-ErbA affects transcription of erythroidspecific genes, therefore blocking differentiation during lineage commitment. Using transient transfection assays in AEV-transformed erythroid progenitor (HD3) cells we have assessed cis-acting sequences governing the CA II-HS2 function and have defined HS2 as a true tissue-specific enhancer that acts independent of its position and orientation with respect to the promoter. Furthermore, the activation of the HS2 enhancer is dependent on factors bound to GATA-protein binding sites and v-ErbA acts by quenching their activity. Our study reinforces that vErbA acts as a dominant negative TR by occluding the VRE and ablating the T3-mediated activation of CA II. However, when the HS2-VRE is linked to the tk promoter, v-ErbA, but not unliganded TR, is inefficient in repressing the transcriptional activity intrinsic to the tk promoter. These data suggest that $\mathrm{v}$-ErbA exerts only part of the full complement of negative, repressive activity intrinsic to TR.

\section{Results}

\section{Carbonic anhydrase II HS2 region acts as a true enhancer}

Using DNase I hypersensitivity mapping, we have previously identified a novel $\mathrm{v}$-ErbA responsive element (VRE), comprised of two half-sites in a direct repeat configuration spaced by four nucleotides (DR4), located in a hypersensitive site in the second intron of carbonic anhydrase II (CA II) gene, termed HS2 (Ciana et al., 1998 and Figure 1a). Transient transfection assays in AEV-transformed HD3 cells using a HS2-containing reporter (HS2-tk) showed that HS2 does not confer any transcriptional activity to the promoter. However, mutation of the first half-site of the VRE (M1-HS2-tk), that abolished v-ErbA binding resulted in a 30-fold enhancement of transcription (Ciana et al., 1998 and Figure 1b). These and other observations suggested that the HS2 region likely functions as an enhancer which is repressed by vErbA.

Operationally, enhancers are defined as cis-acting sequences that activate transcription of promoters in a position- and orientation-independent manner. To test orientation (in)dependency we first placed the M1-HS2 element in both orientations $(+$ and -$)$ upstream of its cognate CA II promoter $(-180$ to +51$)$ yielding M1-HS2(+)-CAII and M1-HS2(-)-CAII constructs, respectively. Transient transfection assays in HD3 cells showed that M1-HS2 boosted the level of transcription via the CA II promoter to the same extent as observed for the tk promoter reporter (Figure 1b). Moreover, the HS2 enhancer acted in both orientations. As expected, the HS2 fragment with the wild type VRE did not activate transcription from the CA II promoter (data not shown). Similar results were obtained with M1-HS2 as an enhancer in combination with a longer CA II promoter fragment $(1.4 \mathrm{~kb}$, position -1362 to +51) (data not shown).

Since the natural position of the HS2 enhancer in CA II gene is in the second intron (Figure 1a), we tested whether HS2 could enhance transcription from a position downstream of the CA II promoter. Transcription of the resulting constructs (CAII-M1-HS2) was boosted 11-16-fold (Figure 1c). Analogously, the M1-HS2 fragment placed in either orientation downstream of the tk promoter also enhanced transcription but to a lesser extent (data not shown).

Taken together, these results unambiguously show that HS2 acts both upstream and downstream of its natural CA II promoter as well as the heterologous tk promoter in an orientation independent fashion and is therefore a bona fide enhancer. Furthermore, v-ErbA appears to impair the activity of the potent HS2 enhancer. 


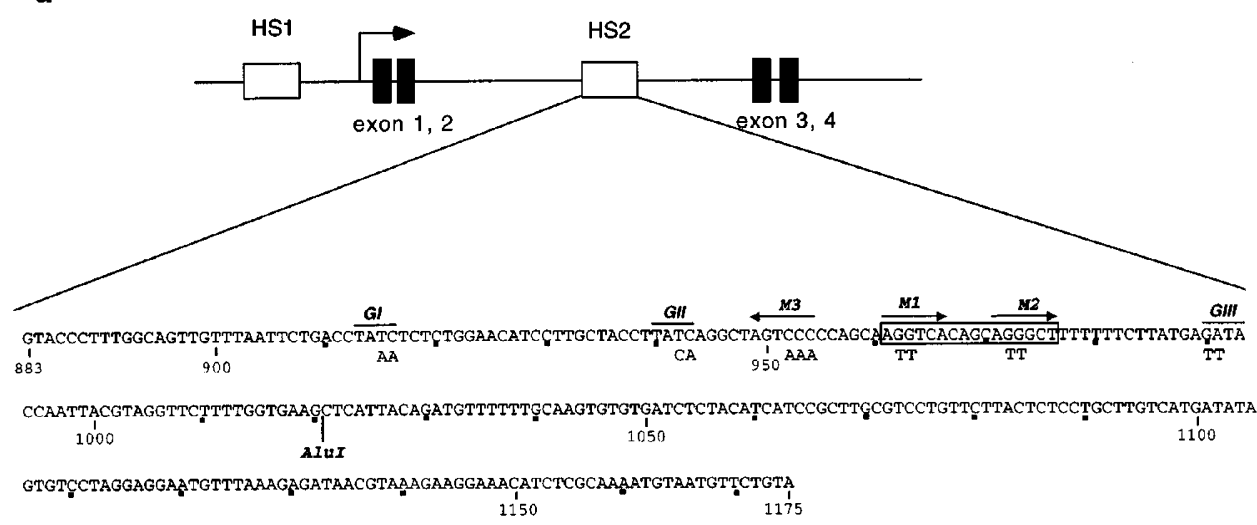

b
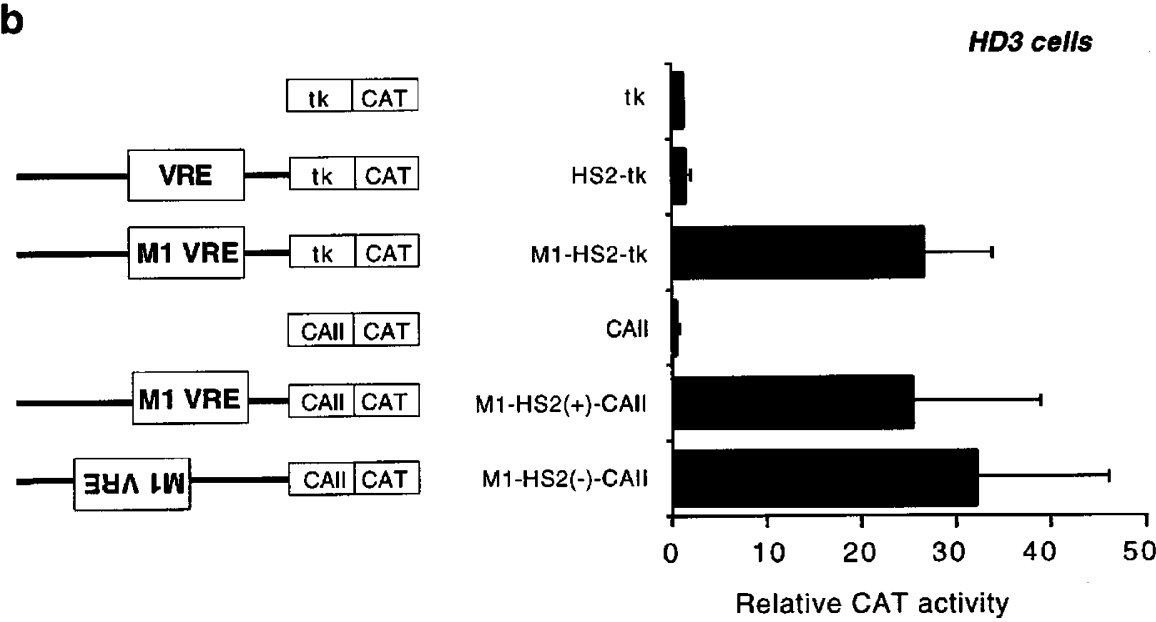

C

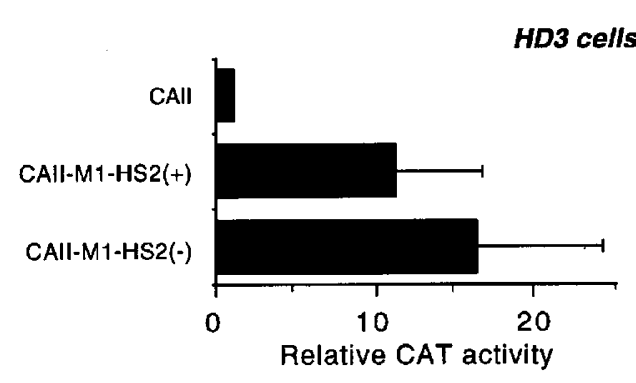

Figure 1 The HS2 fragment functions as a true enhancer (a) Schematic representation of the CA II locus with exons 1-4 (black boxes) and DNase I hypersensitive sites HS1 and HS2 (open boxes). The insert details the 288 bp (RsaI-RsaI) fragment with $A l u \mathrm{I}$ site defining the $3^{\prime}$ boundary of the 135 bp fragment used in most of the following experiments. GI, GII and GIII indicate position of GATA-sites that were mutated and M1, M2 and M3 show the mutated receptor-half-sites. The VRE sequence is boxed. Letters below the sequence indicate the substitution mutations in the various HS2 constructs. (b) Transient transfection assays in HD3 cells with CA II promoter reporter constructs carrying the $135 \mathrm{bp}$ M1-HS2 enhancer fragment in both orientations. The values represent the mean value of the relative chloramphenicol acetyltransferase (CAT) activity, as normalized by luciferase values of the internal control, of at least three experiments, with error bars shown. Transcriptional activity of tk-CAT construct is arbitrarily set to 1 . (c) Transfection assays in HD3 cells with reporters containing the M1-HS2 fragment downstream of the CA II promoter as indicated. Transcriptional activity of CA II reporter is set to 1

\section{Delineation of the HS2 enhancer}

To dissect the molecular mechanism of repression by the v-ErbA oncoprotein, we set out to identify cisacting sequences that are important for the observed HS2 enhancer activity. Given the presence of high levels of v-ErbA in HD3 cells, fragments spanning the HS2 and carrying the wild type VRE sequence do not display enhancer activity (Ciana et al., 1998 and Figure 1b). Therefore, we constructed a series of $5^{\prime}$ and $3^{\prime}$ deletion mutants within the context of M1(883-1175)tk, a 288 bp fragment encompassing the entire HS2, to screen for cis-acting sequences (Figure 2). This construct showed enhancer activity comparable to that of the previously analysed, $135 \mathrm{bp}$ containing M1-HS2tk reporter. Two deletions from the $5^{\prime}$ end of the 
M1(883-1175)-tk fragment significantly reduced the HS2 activity (Figure 2), while further deletion including the VRE (plasmid HS2(979-1175)-tk) resulted in abolishment of the enhancer activity.

Analysis of a $3^{\prime}$ deletion mutant of M1-HS2-tk (up to position -983) revealed a transcriptional activity similar to M1-HS2-tk, while further $3^{\prime}$ deletion including the VRE (up to position -958) resulted in slightly lower levels of transcription. Taken together, our data show that sequences critical for the enhancer activity are located mostly upstream of the VRE. For reasons of consistency, the $135 \mathrm{bp}$ HS2 region used in our previous study (Ciana et al., 1998), which exhibits the full enhancer activity in HD3 cells, was used in the remainder of the experiments.

\section{HS2 is an erythroid-specific enhancer}

To assess whether HS2 acts as a ubiquitous or cell typespecific enhancer we performed transfection experiments using the HS2-tk and M1-HS2-tk reporters in a number of different erythroid and non-erythroid cells (Figure 3). In normal, untransformed, TGF $\alpha / T \mathrm{GF} \beta$ induced chicken Erythroid Cells, T2EC (Gandrillon et al., 1999), in mouse erythroleukemia (MEL) cells as well as in human chronic erythroleukemia cell line K562, HS2 boosted transcription seven- and 20-fold, respectively. In T2ECs and MEL, the mutated M1-HS2 enhancer displayed higher transcriptional activity as compared to the wild type HS2 (13- and 20-fold, respectively) whereas in $\mathrm{K} 562$, the activity of the M1HS2-tk was twofold reduced. The higher level of transcription obtained with $\mathrm{M} 1-\mathrm{HS} 2$ as compared to the parental HS2 in T2ECs and MEL cells suggested that the introduced mutations might have disrupted a binding site for a cellular repressor distinct from vErbA. The HS2(883-958)-tk reporter in which the VRE and surrounding sequences were deleted displayed a much higher activity in T2ECs as compared to M1HS2- and HS2-tk. This would suggest that the M1 mutation of the consensus nuclear receptor half-site did not (fully) abolish repression mediated by sequences in the 958-1020 region. In MEL cells, however, the HS2(883-958) enhancer fragment displayed transactivation activity similar to HS2. Because the HS2(979$1175)$-tk reporter did not display transcriptional activation in MEL cells (data not shown), it seems likely that the M1 mutation had fortuitously created a binding site for a weak activator present in MEL cells. In contrast, in K562 the HS2(883-958) and the M1-HS2 enhancers were equally active but reduced as compared to the parental HS2 suggesting that the HS2 enhancer may be activated by an endogenous nuclear receptor. Neither the parental nor the variants of the HS2 enhancer displayed transcriptional activity when transfected in the non-erythroid chicken embryonic fibroblasts (CEF) and mouse fibroblasts (L) cells (Figure 3). Taken together our data suggest that the HS2 enhancer activity is confined to erythroid cells. The different transcriptional activities of HS2 and M1-HS2 in the various cells types appears to be dependent on the differentiation stage of the erythroid cells or on species specificity.

\section{GATA-factors binding sites contribute to the HS2 enhancer activity}

In HD3 cells the obtained overall activity of the HS2 enhancer probably is the net result of repression
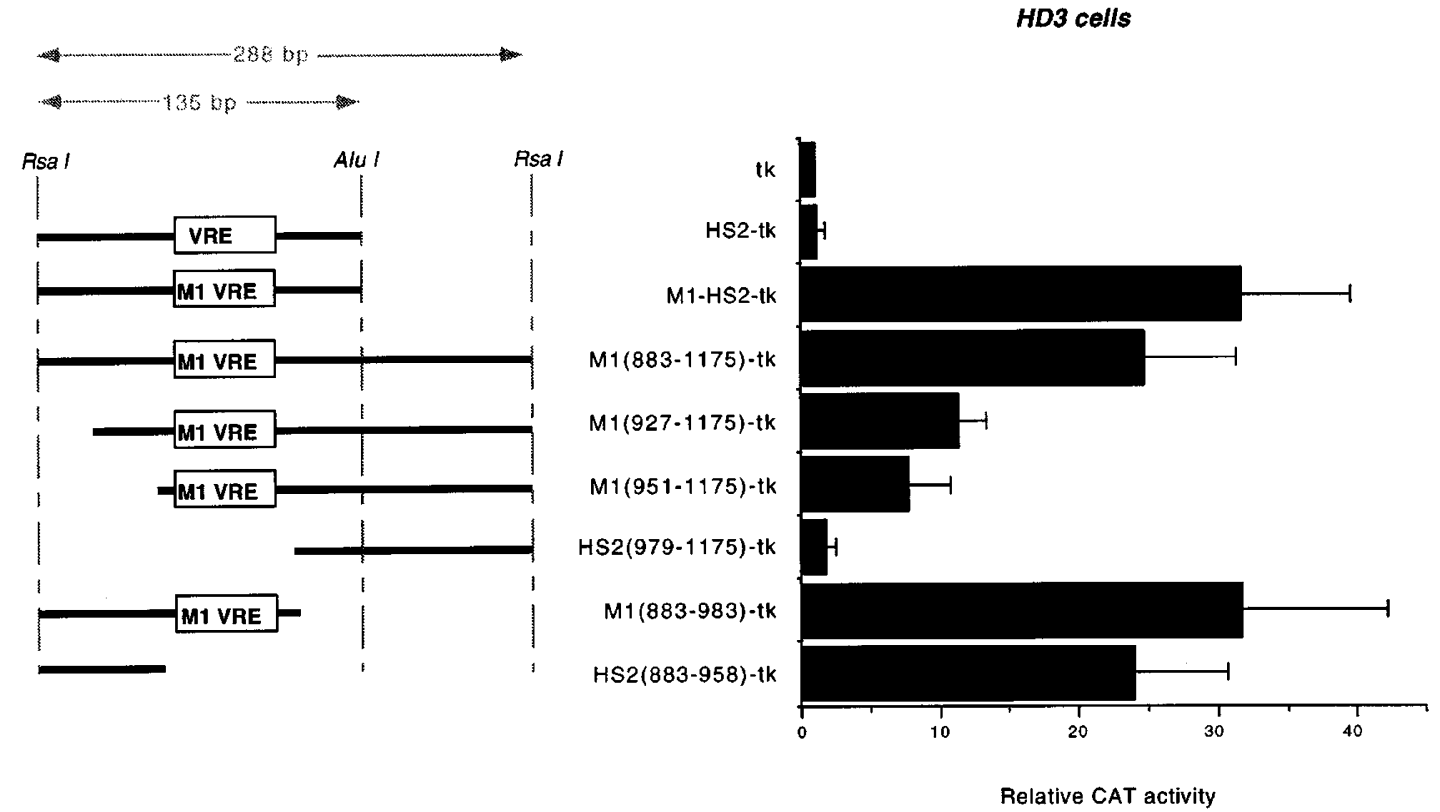

Figure 2 Delineation of the intronic enhancer region HS2 of the CA II gene. Assays of CAT expression of HD3 cells transfected with the indicated fragments of HS2 enhancer cloned in front of tk promoter. Transcription is expressed relative to tk promoter alone 

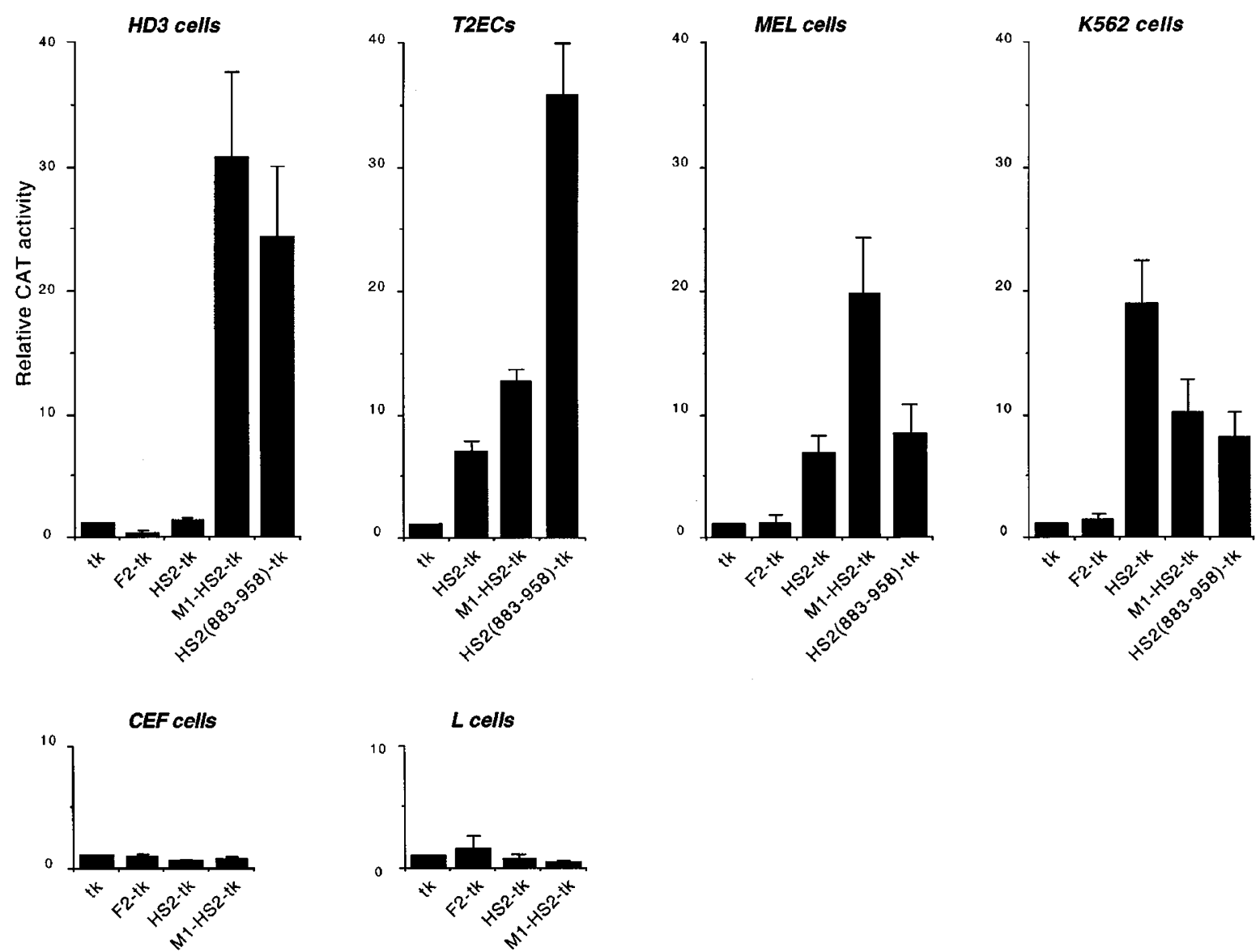

Figure 3 The HS2 enhancer activity is erythroid-specific. Transient transfection assays with the indicated tk reporter constructs in various cell lines as shown. The values represent the mean value of the relative CAT activity of at least three experiments. Transcriptional activity of tk-CAT construct is arbitrarily set to 1

instigated from the VRE and enhancement from positive (erythroid-specific) factors binding to the enhancer. We have previously shown (Ciana et al., 1998) that two of the three potential GATA-factor binding sites present in the HS2 enhancer fragment (positions 913 and 940 referred to as I and II, respectively) (Figure 1a) were protected in DMS footprint assays in vivo and showed DNase I hypersensitive cutting in vitro. A third potential GATA-site (III, position 990) was protected only in in vitro footprint assays. Using electrophoretic mobility shift assays (EMSA), we also showed that the GATA-1 protein present in HD3 cells binds to GATA-site I. Transient transfection assays confirmed that this GATA-element was required for the HS2 enhancer activity.

To elucidate the function of the two other GATAsites, we introduced mutations in these sites in the M1HS2-tk context i.e. in a configuration in which the contribution of the GATA-sites can be monitored. In transient transfection assays in HD3 cells, mutation of either of the GATA-sites I or II (GI-M1-HS2 and GIIM1-HS2, respectively) abolished enhancer activity (Figure 4). Similar results were obtained in K562 and MEL cells (data not shown). In contrast, mutation of the GATA-site III (GIII-M1-HS2) resulted only in a moderate reduction of the enhancer activity. These results are in accordance with the deletion analysis (Figure 2) showing that DNA sequences downstream of the VRE do not significantly contribute to the overall activity of the enhancer. In conclusion, the HS2 enhancer activity is strongly dependent on the two adjacent GATA-factor binding sites (I and II) located upstream of the VRE, suggesting that v-ErbA quenches the positive transcriptional activity of factors (possibly GATA-proteins) bound to these GATA-sites.

\section{HS2 contains multiple half-sites}

We have previously shown that $\mathrm{v}$-ErbA binds to the VRE as a heterodimer with RXR (Ciana et al., 1998). The HS2-VRE deviates from a canonical TRE element in the second half-site, an $\mathrm{AGGgCt}$ sequence rather than AGGTCA (Figure 1a). Closer inspection of the HS2 sequence revealed additional potential nuclear receptor half-sites. Particularly, the non-consensus half-site (position 949-954) located six base-pairs upstream of the VRE together with the first half-site of the VRE forms an everted repeat (ER) spaced by six nucleotides (Figure 1a). ER6-type elements, such 


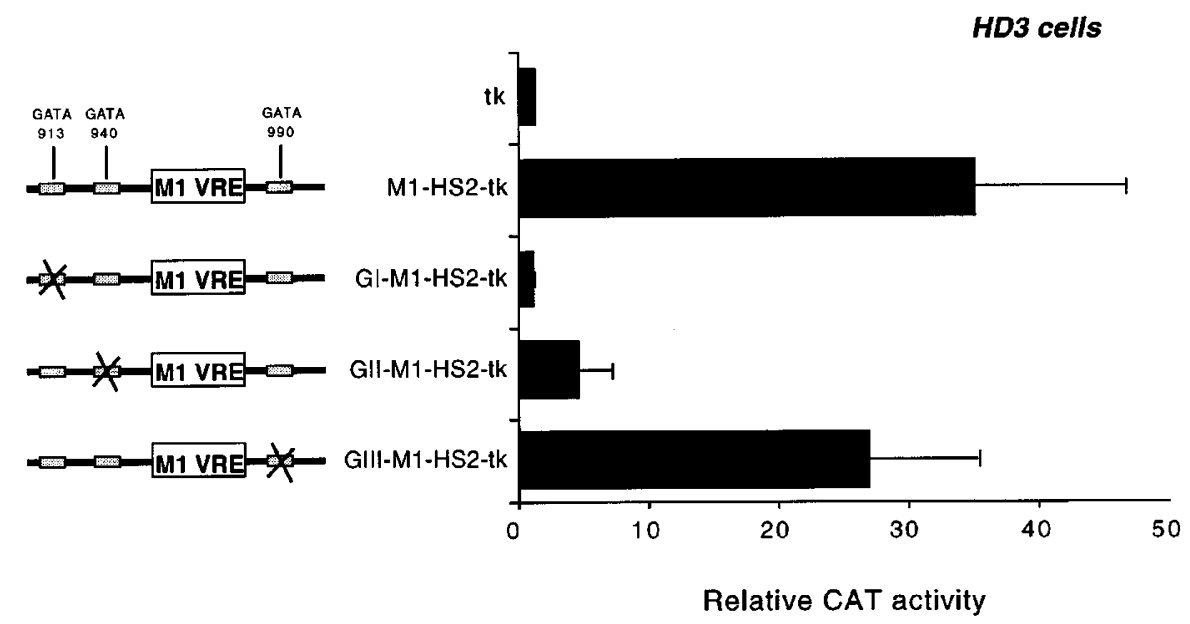

Figure 4 GATA-factor binding sites are required for the HS2 enhancer activity. Transient transfection assays in HD3 cells with tk reporters carrying the M1-HS2 fragment with mutations of the individual GATA-sites as shown on the left. The values represent the mean value of the relative CAT activity of at least three experiments. Transcriptional activity of tk-CAT construct is arbitrarily set to 1

as the F2 from the chicken lysozyme promoter, have been shown to be targets for v-ErbA regulation of transcription (Baniahmad et al., 1990; Wahlstrom et al., 1996; Subauste and Koenig, 1998). Therefore we investigated the relative contribution of each of these half-sites in the v-ErbA mediated transcriptional repression via HS2 using mutagenesis analysis (Figure 5). Transient transfection assays of the resulting M2HS2-tk and M3-HS2-tk constructs in HD3 cells revealed enhancement of HS2 transcription suggesting that both the DR4 and the ER6 elements equally contribute to repression of the enhancer activity. Furthermore, we observed that the central consensus half-site alone was sufficient to confer repression (twofold) (Figure 5), consistent with data from Baniahmad et al. (1990). Thus, all three half-sites contribute to the $\mathrm{v}$-ErbA-mediated repression, but only the central consensus half-site is critical.

\section{VRE can act as a TRE}

Given the altered DNA binding specificity of v-ErbA due to the P-box mutation (Subauste and Koenig, 1995; Judelson and Privalsky, 1996; Wahlstrom et al., 1996), it was not a priori granted that TR could bind to the CA II-VRE and mediate T3-dependent activation. We, therefore, performed EMSA experiments using the VRE oligonucleotide and extracts from HD3 cells or nuclear receptors expressed from vaccinia virus. As shown in Figure 6a, RXR-TR efficiently bound to the VRE (lane 8) suggesting that this element may serve as a TRE. Neither v-ErbA nor RXR alone bound efficiently to the VRE under these conditions (lanes $3-5$ ), while their combination (lanes 6 and 7) led to formation of a protein-DNA complex with mobility similar if not identical to that obtained with HD3 cell extracts (lane 2). The faster migrating complex (lanes 3 , 4, 6 and 7) most likely represents a v-ErbA monomer bound to the VRE.
To measure the relative affinities of RXR-v-ErbA and RXR-TR for the VRE, off-rate experiments were performed. Addition of unlabelled VRE probe as a competitor led to the immediate disappearance of the RXR-v-ErbA complex, whereas RXR-TR was detectable up to 4 minutes after addition of the competitor (Figure $6 \mathrm{~b}$ and data not shown). The $\mathrm{t}_{1 / 2}$ for the RXR$\mathrm{v}$-ErbA complex on the VRE is in the range of seconds while that for RXR-TR is in the range of minutes, showing that the affinity of RXR-v-ErbA for the HS2VRE is significantly lower than that of RXR-TR. A gag-TR $\alpha$ receptor fusion (termed V3) expressed in the HD3V3 cells (Disela et al., 1991) appeared to bind to the HS2-VRE in conjunction with endogenous RXR with kinetics similar to that of recombinant RXR-TR (data not shown). These observations suggest that the differential binding behaviour of RXR-v-ErbA and RXR-TR was not due to an intrinsic difference in DNA binding characteristics between recombinant, vaccinia expressed and 'natural' receptors. It should be noted that the RXR-TR complex formed on the MoMLV-TRE (DR4-type) or on a consensus DR4 is more stable than on the VRE (Bugge et al., 1992 and data not shown), emphasising that the VRE is not an optimal binding site for RXR-TR.

To investigate whether the HS2-VRE can mediate T3-induced activation, we performed transient transfection assays in HD3V3 cells in which the level of expression of gag-TR $\alpha(\mathrm{V} 3)$ is comparable to that of $\mathrm{v}$ ErbA (Disela et al., 1991). Transfection of the HS2-tk reporter construct in these cells yielded transcription levels comparable to those obtained in HD3 cells, thereby suggesting that HS2 enhancer activity can be quenched by gag-TR $\alpha$. Addition of T3 boosted the level of transcription 4.5 -fold, which is only $25 \%$ of the activity obtained with M1-HS2-tk in these cells (Figure 6c and Ciana et al., 1998). The impaired ability of the wild type HS2 enhancer to display full activity in response to T3 is most likely due to the high levels of 
HD3 cells

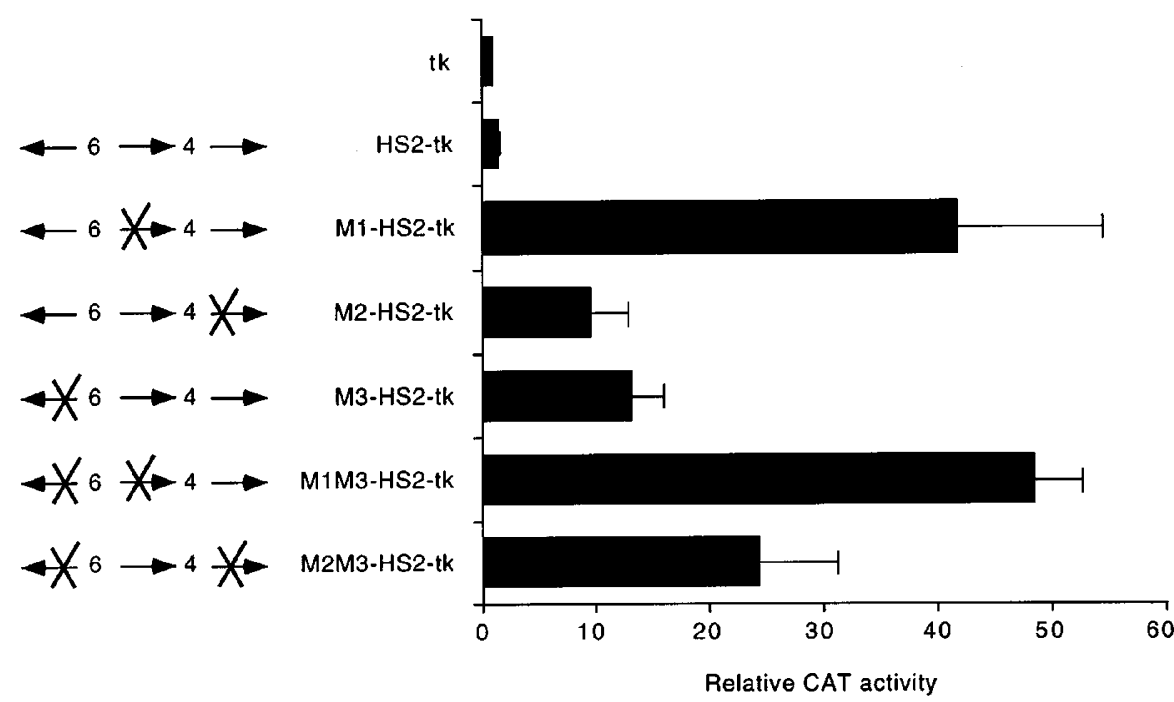

Figure 5 The HS2 enhancer contains multiple half-sites that can mediate repression by v-ErbA. Transient transfection assays in HD3 cells with tk reporters containing mutations of the indicated potential half-sites (see Figure la for sequence). The values represent the mean value of the relative CAT activity of at least three experiments. Transcription is expressed relative to tk promoter alone

v-ErbA in HD3V3 cells opposing the activation by ligand-bound RXR-V3.

To directly compare the function of the VRE with that of a canonical TRE in the context of HS2 enhancer, we replaced the VRE with a consensus DR4 element by changing the $3^{\prime}$ most half-site of the VRE (position 971-976) into AGGTCA yielding DR4-HS2. In HD3V3 cells and in the absence of hormone, transcription of the DR4-HS2-tk construct was repressed to levels similar to that obtained with HS2-tk suggesting that unliganded v-ErbA and/or V3 quenches the enhancer activity via a DR4. Administration of $\mathrm{T} 3$ resulted in elevated levels of activation as compared to HS2-tk (seven- and 4.5-fold, respectively) (Figure 6c). To further investigate the ability of the VRE to mediate a T3-response, we tested the minimal VRE sequence placed in front of the tk promoter in HD3V3 cells (VRE-tk). T3-dependent transcriptional activation of the VRE-tk was merely 1.7-fold, while of the F2 element and of the well-characterised MoMLVTRE (Sap et al., 1989) was six- and eight-fold, respectively (Figure 6c, Ciana et al., 1998 and data not shown). In summary, these data show that thyroid hormone responsiveness can be mediated by the VRE in the HS2 context. DR4, however, is more potent since it nearly restores enhancer activity as defined by the M1-HS2. Secondly, the VRE by itself possess very little activation potential in cells that co-express vErbA and gag-TR $\alpha$.

\section{$v$-ErbA and gag-TR $\alpha$ act differently to instigate repression via the HS2 enhancer}

Unliganded TR and v-ErbA are thought to repress transcription of T3-regulated genes. Support for this model is provided by experiments using the $\mathrm{F} 2$ element from the chicken lysozyme gene promoter; transcription of the F2 element is repressed 6-7-fold as compared to tk alone in HD3 cells (Baniahmad et al., 1992; Ciana et al., 1998 and Figure 3). We have so far shown that the wild type HS2 enhancer is 'neutral', i.e. it neither enhances nor represses transcription from the homologous or from a heterologous promoter in HD3 cells. This suggests that positive and negative factors regulating its activity are in balance. To investigate whether v-ErbA could actively repress the transcriptional activity of the heterologous tk promoter we mutated GATA-sites I and II within the wild type HS2 enhancer so that the negative transcriptional activity of v-ErbA would prevail (Figure 7a). Surprisingly, transfection experiments in HD3 cells showed that mutation of these GATA-sites (GI-HS2-tk and GII-HS2-tk reporters) did not reduce transcription significantly below that of the enhancerless tk promoter (less than twofold) (Figure 7a). These data suggest that in HD3 cells v-ErbA does not efficiently, if at all, repress the transcriptional activity intrinsic to the heterologous tk promoter.

To test whether the incapacity of v-ErbA to convey transcriptional repression to the linked tk promoter was an intrinsic property of $\mathrm{v}$-ErbA and/or dictated by the VRE element, similar experiments were performed in HD3V3 cells that express equivalent levels of gag-TR $\alpha(\mathrm{V} 3)$ and v-ErbA. In HD3V3 cells, the levels of transcription of GI-HS2-tk and GII-HS2tk were strongly repressed as compared to HS2-tk and more importantly to tk alone (six- and 3.5-fold, respectively) (Figure 7a). An even more pronounced effect was obtained when a DR4-HS2 derivative carrying a mutation in the GATA-site I was tested. 
a

\begin{tabular}{|l|l|l|l|l|l|l|l|l|}
\hline HD3 extract & & + & & & & & & \\
\hline vv. V-ErbA & & & ++ & + & & ++ & + & \\
\hline vv. RXR & & & & & + & + & + & \\
\hline vv. RXR-TR & & & & & & & & + \\
\hline
\end{tabular}

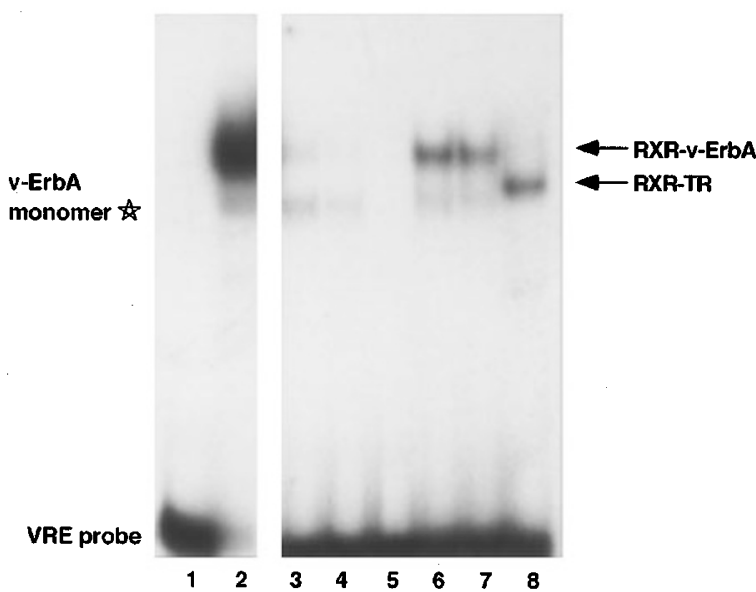

C

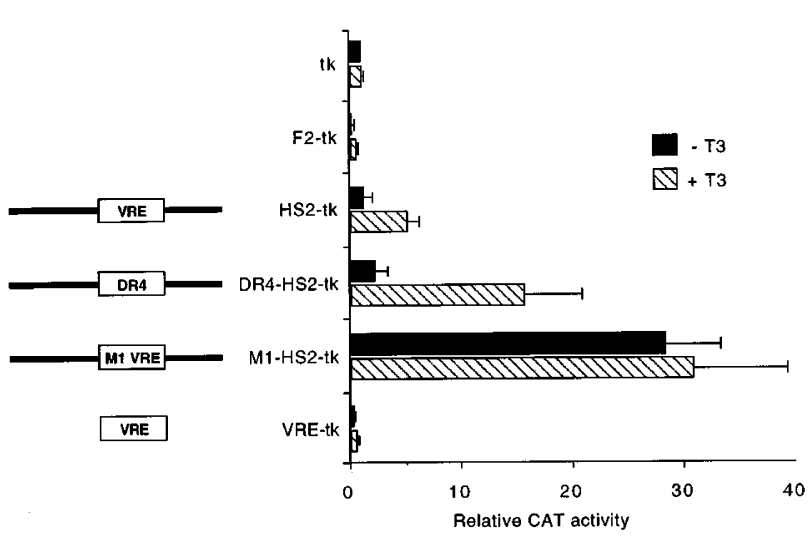

Figure 6 Liganded TR binds to and activates transcription from the HS2-VRE. (a) A gel mobility shift assay of ${ }^{32} \mathrm{P}$-labelled synthetic oligonucleotide containing the VRE sequence using HD3 cell-extract or vaccinia-expressed receptor proteins, as indicated. (b) Off-rate assay performed with the same probe as in a and either HD3 cell-extract or vaccinia-expressed RXR-TR. Lane 1: free probe; lane 2: VRE probe plus HD3 cell-extract; lanes 3-7: as in lane 2 plus 1000-fold of unlabelled VRE probe added as competitor, with aliquots loaded on the gel at the indicated time points; lane 8: VRE probe plus vaccinia-expressed RXR-TR; lane 9-13: as in lane 8 plus unlabeled VRE probe as competitor with aliquots loaded on the gel at the indicated time points. (c) Transient transfection assays in HD3V3 cells, expressing gag-TR $\alpha$ and v-ErbA, with the indicated tk reporter constructs. Black bars denote relative CAT activities in the absence of hormone while hatched bars represent CAT values in the presence of T3. Transcription is arbitrarily set to 1 for the to tk promoter alone in the absence of hormone

The GI-DR4-HS2-tk reporter yielded a 10-fold repression of transcription as compared to tk alone in HD3V3 cells whereas the repression was less than twofold in HD3 cells. Finally, in HD3 cells the minimal HS2-VRE element (VRE-tk) yielded 1.3-fold repression of transcription as compared to tk whereas in HD3V3 cells the repression was up to fivefold. Taken together these results show that v-ErbA in contrast to gag-TR $\alpha$ is unable to convey repression to the tk promoter when bound to the HS2-VRE. This differential activity is dictated by both the HS2 context and the VRE since transcription of the F2- tk is equally repressed in both cell lines (Figure 7a). Taken together, our data indicate that the ability of the $\mathrm{v}$-ErbA oncoprotein to repress is not equivalent to that of unliganded gag-TR $\alpha$.

\section{Discussion}

In this study we have continued to probe the function of the leukemia inducing oncoprotein, v-ErbA, in the control of transcriptional repression. Our detailed analysis of the previously identified regulatory region 

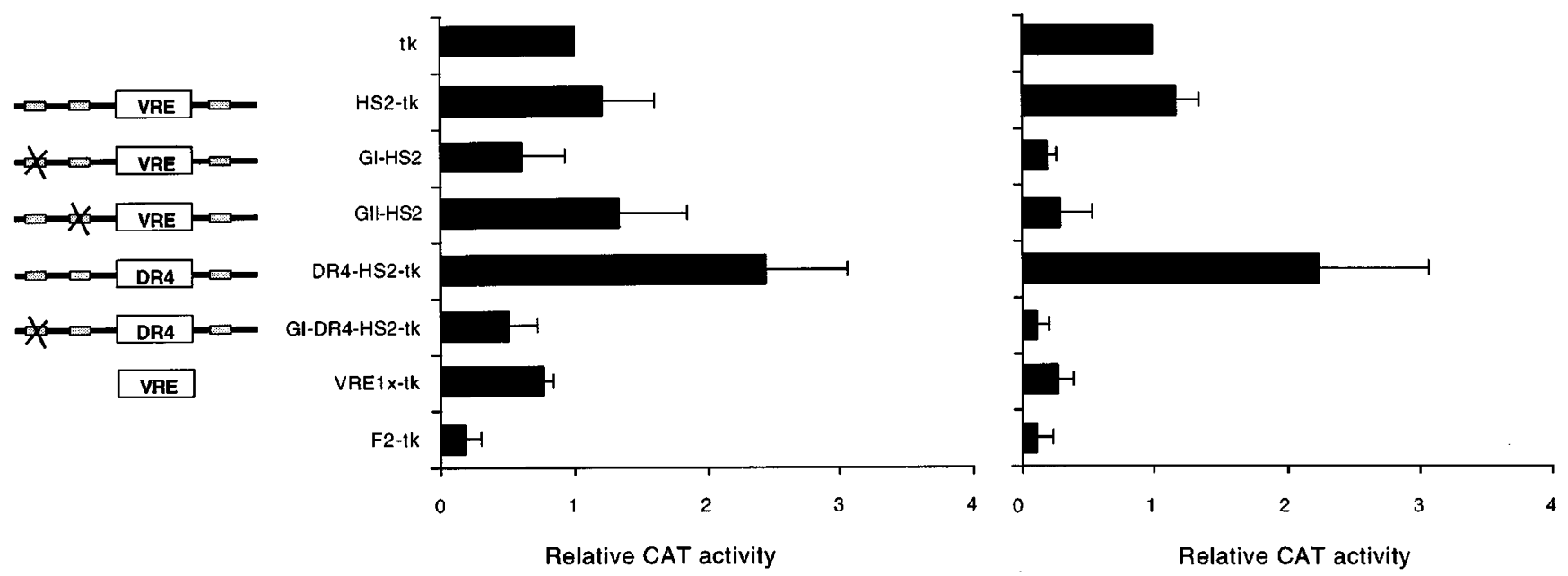

b

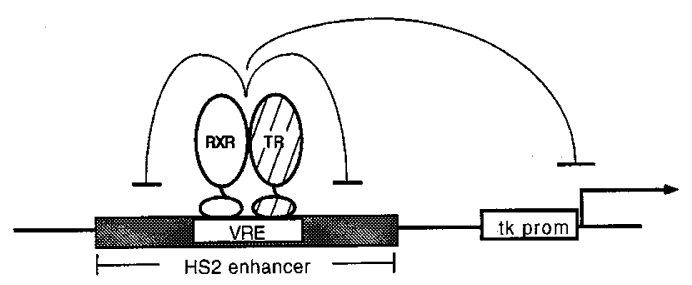

Unliganded TR

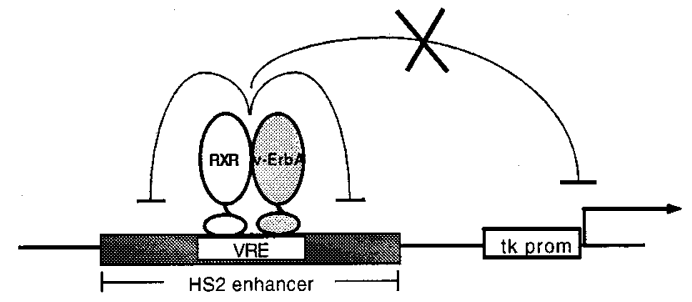

v-ErbA

Figure 7 v-ErbA, unlike unliganded gag-TR $\alpha$, cannot repress the transcriptional activity intrinsic to the tk promoter, when bound to the HS2-VRE. (a) Transient transfection assays in HD3 cells (left) and in HD3V3 cells (right) with the indicated tk reporters. The values represent the mean value of the relative CAT activity of at least three experiments. Transcription is expressed relative to tk promoter alone for both cell lines. (b) Proposed model whereby unliganded gag-TR $\alpha$ can both quench the HS2 enhancer and the transcription from tk promoter whereas the v-ErbA oncoprotein can only quench the activity of the enhancer

in the erythroid stage-specific CA II gene (Ciana et al., 1998) now provides a more precise model for the vErbA-mediated repression. Firstly, we have shown that the regulatory region of the CA II gene located in the second intron, termed HS2, is a true enhancer that can activate transcription independently of its position and orientation relative to the promoter. The apparent erythroid-specific activity of the enhancer in primary erythroid progenitors as well as in other erythroid cells appears to be dictated primarily by two adjacent GATA-factor binding sites. Since both v-ErbA and unliganded TR can efficiently quench the activity of the HS2 enhancer it appears that they both interfere with the positive function of the erythroid GATA-factors. Secondly, we have shown that the HS2 enhancer can convey T3-mediated activation to a heterologous or CA II promoter (Figure 6c and data not shown), providing additional evidence for the role of $\mathrm{T} 3$ in the activation of CA II transcription (Disela et al., 1991). Thirdly, we have provided evidence that $v$-ErbA bound to VRE, in contrast to unliganded TR, is impaired in repressing the tk promoter.
Mutation and deletion analyses (Figures 3 and 4) showed that the HS2 enhancer activity is greatly dependent on two GATA-factor binding sites and that v-ErbA when bound to the VRE can quench their positive transcriptional activity. Interference of nuclear receptor superfamily members with the function of GATA-factors appears to be a recurring theme. For example, the promoter of the slow myosin heavy chain (MyHC) 3 gene contains one GATA-site and one Vitamin D3 responsive element (VDRE). While GATA-factors enhance the transcriptional activity of the MyHC 3 promoter, VDR binding leads to transcriptional repression in ventricular cardiomyocytes (Wang et al., 1998). Moreover, liganded glucocorticoid receptor (GR) has been shown to inhibit the function of GATA-1 protein on the promoters of the $\beta$-major and $\beta$-minor globin genes, most probably via a direct interaction between GATA-1 and GR (Chang et al., 1993). In pilot immunoprecipitation experiments, we did not observe a direct interaction between GATAfactors and v-ErbA, whereas the interaction between vErbA and NCoR could readily be detected (GG 
Braliou and HG Stunnenberg unpublished observations).

The presence of enhancers in introns, regulating transcription of tissue-specific genes, such as the HS2 from the CA II gene is not uncommon. Examples in the hematopoietic system are the $3^{\prime}$ enhancer of the chicken adult $\beta$-globin and the intronic immunoglobin enhancer $(\mathrm{E} \mu)$ (Emerson et al., 1987; Jenuwein et al., 1997). The $3^{\prime}$ intronic enhancer of the chicken adult $\beta$ globin gene binds GATA-factors that could co-operate with GATA-factors bound to a site proximal to the TATA box to instigate transcription (Emerson et al., 1987). We speculate that a similar mechanism may be in place for the CA II locus since the CA II promoter contains a GATA-motif. Our findings that HS2 can enhance transcription from its own CA II promoter more efficiently compared to the heterologous tk promoter, is in line with this model. Further investigations are required to address the nature of the interference of v-ErbA with GATA-factors activity on the CAII-HS2 enhancer.

Using normal, untransformed chicken erythroid progenitors (T2ECs) (Gandrillon et al., 1999), HD3, MEL and K562 cells as well as non-erythroid CEF and L cells, we have shown that the HS2 enhancer activity is erythroid-specific (Figure 3). The erythroidspecific GATA-factors appear to govern the transcriptional activity of the enhancer (Figure 4 and data not shown). It is also evident that $v$-ErbA accounts for the repression of HS2 enhancer in HD3. This notion is further corroborated by the observation that cotransfection of $\mathrm{v}$-ErbA in both MEL and K562 cells diminished the transcriptional activity of HS2 (data not shown). The variation in the transcriptional activities as obtained with HS2, M1HS2 and HS2(883-958) is probable due to differences in the concentration of transcription factors present at the different stages of erythroid differentiation in which the HD3, MEL, K562 cells and T2ECs are arrested. The higher activity of the HS2(883-958) enhancer as compared to HS2 and M1-HS2 in T2ECs can be taken as evidence for the presence of a cellular repressor in early erythroid progenitors. It is tempting to speculate that the $\mathrm{v}$-ErbA oncoprotein fulfills the role of the cellular repressor in transformed HD3 cells in repressing the CA II and other targets.

In previous studies we and others showed that $\mathrm{TR} \alpha$ is likely to be involved in erythroid differentiation (Disela et al., 1991; Schroeder et al., 1992; Gandrillon et al., 1994). We showed that liganded $\mathrm{TR} \alpha$ plays a role in CA II activation presumably by increasing the accessibility of the HS2 (Ciana et al., 1998). The notion that $\mathrm{v}$-ErbA occludes liganded TR from its binding site is supported by the fact that substitution of the VRE with a canonical DR4, a better binding site for TR, potentiates the T3 response (Figure 6c). Therefore, the sequence composition of the VRE is critical for the efficiency of the occlusion since v-ErbA occludes liganded gag-TR $\alpha$ more efficiently on the VRE than on the DR4.
As mentioned before, v-ErbA prevents the HS2 enhancer by quenching the GATA-factors activity, a function that is exerted locally within the HS2 region. Gag-TR $\alpha$ can also quench HS2 activity (Figure 7a, compare HS2-tk in HD3 and HD3V3 cells) consistent with the observation that in erythroid cells overexpressing gag-TR $\alpha$ CA II is repressed in the absence of T3 (Disela et al., 1991; Bauer et al., 1998). However v-ErbA, unlike unliganded gag-TR $\alpha$, was unable to actively repress transcription from the heterologous tk promoter when bound to the HS2-VRE (Figure 7a). Unliganded $\operatorname{TR} \alpha$ and $\operatorname{TR} \beta$ have been reported to interfere with the preinitiation complex (PIC) assembly to actively repress transcription. It has been shown that TR directly interacts with TFIIB (Baniahmad et al., 1993; Fondell et al., 1993, 1996) while v-ErbA is impaired in such an interaction (Urnov et al., 2000). We speculate that $v$-ErbA is unable to interact with TFIIB in the context of the HS2-VRE, and thus cannot interfere with factors governing the basal transcription of the tk promoter.

Recent data have implicated helix 12 (H12) of the receptors LBD in corepressor binding (Zhang et al., 1999; Renaud et al., 2000). Given that v-ErbA lacks an intact $\mathrm{H} 12$, it is possible that distinct binding affinities for corepressors might account at least partially for the differential v-ErbA and TR repression capacity. Our finding that $\mathrm{v}$-ErbA and TR show distinct repression activities on the VRE but not on the F2 element are consistent with observations suggesting repression and corepressor release to be dependent on the architecture of TREs (Olson et al., 1998; Wahlstrom et al., 1998). Extrapolation of our observations should be done with great care, because the data presented here is based on transient transfection assays where the introduced DNAs adopt an abnormal nucleosomal structure that differs from that of endogenous genes (Archer et al., 1992; Cereghini and Yaniv, 1984; Innis and Scott, 1983; Jeong and Stein, 1994; Reeves et al., 1985; Van Lint et al., 1996). It is throughout possible that v-ErbA utilises additional mechanisms to repress transcription in the context of chromatin. Additional in vivo and in vitro experiments are needed to unravel how v-ErbA functions within chromatin to shed light on the correlation between repression by v-ErbA and its oncogenicity.

In conclusion, v-ErbA appears to regulate transcription in at least three ways: firstly, by quenching the activity of GATA-factors bound to the HS2, secondly, by occluding liganded $T R \alpha$ and thirdly by interfering with the repression function of unliganded TR $\alpha$ (Figure 7b). Similar mechanisms might be in place for other erythroid v-ErbA target genes. We propose that vErbA, apart from the already known ability to occlude liganded TR, can antagonise the function of unliganded $\mathrm{TR} \alpha$ as well when bound to the HS2-VRE, thus preventing the full silencing function of $\mathrm{TR} \alpha$. Altogether, our data fit within an increasing amount of reports demonstrating the existence of subtle differences between the unliganded $\mathrm{TR} \alpha$ and the $\mathrm{v}$-ErbA oncoprotein, in terms of subcellular localization 
(Boucher et al., 1988), competition for a transcriptional repressor (Rascle et al., 1994), binding of cofactors (Barettino et al., 1993), and DNA binding specificity (Chen et al., 1993; Subauste and Koenig, 1995; Judelson and Privlasky, 1996). It is quite possible that these functional differences in the repressive, unliganded modes of $\mathrm{v}$-ErbA and gag-TR $\alpha$ may contribute to v-ErbA oncogenic activity. Nevertheless, the precise contribution of those differences to the transforming ability of v-ErbA will await the yet-to-come functional identification of the transformation-relevant target genes of v-ErbA.

Studies on v-ErbA function are moving our knowledge forward to unravelling the connections between transcriptional repression and oncogenicity. Applying this knowledge will certainly provide novel insights into the molecular mechanisms controlling normal and abnormal erythroid differentiation.

\section{Materials and methods}

\section{Cell culture}

The derivatives of the AEV-transformed cell line HD, HD3EpoR and HD3-V3, expressing the murine erythropoietin receptor or a gag-chicken $\mathrm{TR} \alpha$ fusion, respectively, were grown in CFU-E medium (Dolznig et al., 1995). Before T3 treatment, HD3-V3 cells were grown for $48 \mathrm{~h}$ in medium containing stripped serum using the anion-exchange resin $\mathrm{AG}$ 1-X8 (BIORAD). Chicken embryo fibroblasts (CEF) were grown in Iscoves' medium (Gibco-BRL) supplemented with $8 \%$ fetal calf serum, $2 \%$ chicken serum and antibiotics. MEL cells were grown in DMEM (Gibco-BRL) supplemented with $10 \%$ fetal calf serum and non-essential amino-acids (Philipsen et al., 1990). L cells and were grown in DMEM (Gibco-BRL) supplemented with $10 \%$ fetal calf serum (Baniahmad et al., 1992). K562 cells were grown in RPMI1640 supplemented with $10 \%$ fetal calf serum (Geijtenbeek et al., 2000). T2ECs were grown in LM1 medium as previously described (Gandrillon et al., 1999).

\section{Transient transfection assays}

HD3-EpoR, HD3-V3 and K562 cells were transfected using the DEAE-dextran transfection procedure as previously described (Choi and Engel, 1988). In a typical experiment, $10^{7}$ or $5 \times 10^{6}$ for K562) cells were transfected with 5 (or 10 ) $\mu \mathrm{g}$ of reporter construct together with $1 \mu \mathrm{g}$ of EF $1 \alpha$-Luc as internal control, and harvested after $48 \mathrm{~h}$. One hundred and fifty nM T3 was added to HD3-V3 transfected cells for the last $24 \mathrm{~h}$ where indicated. CAT and luciferase activities were measured as described previously (Barettino et al., 1993).

MEL cells were transfected by electroporation as described (Philipsen et al., 1990). Briefly $2 \times 10^{7}$ cells were incubated on ice for $10^{\prime}$ with $20 \mu \mathrm{g}$ of reporter construct together with $5 \mu \mathrm{g}$ of EF1 $\alpha$-Luc as internal control and $15 \mu \mathrm{g}$ of empty pSG7 vector to keep the same amount of transfected DNA in all samples. Subsequently, the cells were electroporated in a BIORAD gene pulser at $960 \mu \mathrm{F}$ and $280 \mathrm{~V}$ and after $10^{\prime}$ incubation on ice were plated in $10 \mathrm{ml}$ of complete medium. After $48 \mathrm{~h}$, the cells were harvested for measurement of CAT and luciferase activities.

CEF and L cells were transfected with the calcium phosphate method as described (Berkenstam et al., 1992;
Barettino et al., 1993). Briefly, $3 \times 10^{5}$ cells were transfected with $5 \mu \mathrm{g}$ of reporter construct along with $1 \mu \mathrm{g}$ of EF $1 \alpha$-Luc as internal control and $5 \mu \mathrm{g}$ of empty pSG7 vector. After $16 \mathrm{~h}$ the cells were washed with phosphate buffer saline (PBS) and harvested after $24 \mathrm{~h}$ for measurement of CAT and luciferase activities.

T2ECs were transfected with $5 \mu \mathrm{g}$ of reporter construct along with $2 \mu \mathrm{g}$ of EF $1 \alpha$-Luc using Lipofectamine ${ }^{\mathrm{TM}}$ reagent, as will be described in details elsewhere (M Deguillien, $M$ Morinière, S Dazy, O Gandrillon and F Baklouti; manuscript in preparation). After $48 \mathrm{~h}$, the cells were harvested for measurement of CAT and luciferase activities.

Oligonucleotides used for gel retardation assays and for cloning in $\mathrm{pBLCAT2}$ vector

Oligonucleotides used for gel retardation. Coding strand: VRE: 5'-TCGACCCAGCAAGGTCACAGCAGGGCTTTTTTTC-3', Non coding strand: VRE: 5'-TCGAGAAAAAAAGCCCTGCTGTGACCTTGCTGGG-3'. Oligonucleotides used for mutagenesis: pBL5': 5'-TCCCAGTCACGACGTTGTAAA-3' in pBLCAT2; pBL3': 5'- GTTCGAATTCGCCAATGACAA-3' pBLCAT2; mutagenesis-VRE-M1: 5'-CCCTGCTGTGA $A A$ TTGCTG-3'; mutagenesis-VRE-M2: 5'-AAAGCAATGCTGTGACCTTG-3' mutagenesis-VRE-M3: 5'-TTGCTGGTTTACTAGCCTGATAAGG-3'; mutagenesis-GIGATA: 5'-GTTCCAGAGATTTAGGTCAG-3'; mutagenesis-GII-GATA: 5'-GGACTAGCCTTGTAAGGTA-3'; mutagenesis-GIII-GATA: 5'-CTACGTAATTGGTA $A A$ TCATA3' mutagenesis-VRE-DR4: 5'-CTCATAAGAAAAAATG $A$ CCTGCTGT-3'

Oligonucleotides used for cloning: Upper strand. M1-927945: 5'-CCCAAGCTTATCCTTGCTACCTTATCAG-3' (HindIII site underlined) M1-951-971: 5'-CCCAAGCTTTCCCCCAGCAATTTCACAGCA-3' (HindIII site underlined) 979998: 5'-GCTCTAGAGCTTTCTTATGAGATACCAA-3' (XbaI site underlined); 1019-1041: 5'-GCTCTAGAGCTCATTACAGATGTTTTTTGC-3' (XbaI site underlined); CAII upper: 5'-CTTTGATCTGCGCCTCCA-3'.

Lower strand. 1021 - 1004: 5'-GCTCTAGAGCTTCACCAAAAGAACCTA-3' (XbaI site underlined) M1-983-966: 5'GCTCTAGAGCGAGAAAAAAAGCCCTGCTGT-3' (XbaI site underlined) 958-938: 5'-GCTCTAGATGGGGGACTAGCCTGATAAG-3' (XbaI site underlined) CAII lower: 5'CGGGATCCCGCCAGTGATGGGACCTGGTG-3' (BamHI site underlined).

\section{Gel retardation assay}

Twenty pmoles of labelled VRE-oligonucleotide were incubated with HD3 protein extract $(40 \mu \mathrm{g})$ or vaccinia-virus expressed v-ErbA, TR and RXR proteins for 20' on ice (de Magistris and Stunnenberg, 1988; Stunnenberg et al., 1988). Binding was performed as described (Ciana et al., 1998). Offrate competition was performed by adding a 100 - or 1000 fold molar excess of unlabelled VRE-oligonucleotide and aliquots were applied on pre-cooled and pre-run $0.5 \times \mathrm{TBE}$, $5 \%$ polyacrylamide gels at the indicated time points.

\section{Plasmids}

A $R s a \mathrm{I} / R s a \mathrm{I}$ fragment of $288 \mathrm{bp}$ spanning the HS2 was inserted into EcoRV site of pBlueScript and subsequently subcloned into $\mathrm{SalI} / \mathrm{BamHI}$ sites of pBLCAT2 (Lucknow and Schutz, 1987), yielded plasmid HS2(883-1175)-tk. M1(883$1175)$-tk was generated from HS2(883-1175)-tk by oligonucleotide-directed site-specific mutagenesis (Seraphin and 
Kandels-Lewis, 1996) using the oligonucleotides pBL5' and pBL3' and the mutagenesis oligonucleotide M1-VRE. The HS2-tk construct was generated from HS2(883-1175)-tk by PCR amplification using primers pBL5' and 1021-1004, digested with $S a l \mathrm{I} / X b a \mathrm{I}$ and inserted into $S a l \mathrm{I} / X b a \mathrm{I}$ sites of pBLCAT2. M1-HS2-tk was generated from HS2-tk by oligonucleotide-directed site-specific mutagenesis using the oligonucleotide M1-VRE.

M1(927-1175)-tk and M1(951-1175)-tk constructs were generated by PCR amplification of M1(883-1175)-tk using primers 927-945 and M1-951-971, respectively, along with pBL3', digested with HindIII/XbaI and inserted into HindIII/ $X b a \mathrm{I}$ site of pBLCAT2. HS2(979-1175)-tk construct was generated by PCR amplification of HS2(883-1175)-tk using primers 979-998 and pBL3', digested with $\mathrm{XbaI}$ and inserted into XbaI site of pBLCAT2. PCR amplification of M1-HS2tk with primers pBL5' and M1-983-966 or 958-938, digesting with HindIII/XbaI and subsequent cloning into Hind III/XbaI site of pBLCAT2, yielded plasmids M1(883983)-tk and HS2(883-958)-tk respectively. Annealing of primers VRE-upper and VRE lower and ligation with pBLCAT2 digested with SalI yielded plasmid VRE-tk. F2tk plasmid was a kind gift from Baniahmad et al. (1992).

A CA II promoter fragment was PCR amplified using primers CAII-upper and CAII-lower (has a mutation in the ATG of CA II), digested with ApaI, filled in with Klenow and digested with BamHI. The resulting $263 \mathrm{bp}$ promoter fragment, inserted into $X b a \mathrm{I}$, filled in with Klenow and BamHI sites of pBLCAT3, yielded construct CA II. Digesting the CA II with SalI, filling in with Klenow and ligating

\section{References}

Archer TK, Lefebvre P, Wolford RG and Hager GL. (1992). Science, 255, $1573-1576$.

Baniahmad A, Kohne AC and Renkawitz R. (1992). EMBO $J ., 11,1015-1023$.

Baniahmad A, Steiner C, Kohne A and Renkawitz R. (1990). Cell, 61, 505-514.

Baniahmad A, Ha I, Reinberg D, Tsai S, Tsai MJ and O'Malley BW. (1993). Proc. Natl. Acad. Sci. USA, 90, $8832-8836$.

Bannister AJ and Kouzarides T. (1996). Nature, 384, $641-$ 643.

Barettino D, Bugge TH, Bartunek P, Vivanco Ruiz MD, Sonntag-Buck V, Beug H, Zenke M and Stunnenberg HG. (1993). EMBO J., 12, $1343-1354$.

Barettino D, Vivanco Ruiz MM and Stunnenberg HG. (1994). EMBO J., 13, 3039-3049.

Bauer A, Mikulits W, Lagger G, Stengl G, Brosch G and Beug H. (1998). EMBO J., 17, 4291-4303.

Bauer A, Ulrich E, Andersson M, Beug $\mathrm{H}$ and von Lindern M. (1997). Oncogene, 15, 701-715.

Berkenstam A, Ruiz MM, Barettino D, Horikoshi M and Stunnenberg HG. (1992). Cell, 69, 401-412.

Beug H, Bauer A, Dolznig H, von Lindern M, Lobmayer L, Mellitzer G, Steinlein P, Wessely O and Mullner E. (1996). Biochim. Biophys. Acta., 1288, M35-M47.

Boucher P, Koning A and Privalski ML. (1988). J. Virol., 62, $534-544$.

Bugge TH, Pohl J, Lonnoy O and Stunnenberg HG. (1992). EMBO J., 11, $1409-1418$.

Casini T and Graf T. (1995). Oncogene., 11, 1019-1026.

Cereghini S and Yaniv M. (1984). EMBO J., 3, 1243-1253.

Chang TJ, Scher BM, Waxman S and Scher W. (1993). Mol. Endocrinol., 7, 528-542. with an excised insert from M1-HS2 with SalI/XbaI and filled in with Klenow, yielded plasmids M1-HS2-CAII $(+)$ and M1$\operatorname{HS} 2-\mathrm{CAII}(-)$. For the generation of CAII-M1-HS2 $(+) /(-)$ the M1-HS2 insert was excised from M1-HS2-tk construct with $S a l I / X b a$ I filled in with Klenow, and ligated with CAII plasmid digested with $S m a I$.

M2-HS2-tk and M3-HS2-tk were generated from HS2-tk by oligonucleotide-directed site-specific mutagenesis using the mutagenesis oligonucleotides M2-VRE and M3-VRE respectively. M1M3-HS2-tk and M2M3-HS2-tk were generated from M1-HS2-tk and M2-HS2-tk using the M3-VRE oligonucleotide.

M1-HS2 constructs carrying mutations of the GATA-sites were generated from M1-HS2-tk and mutagenesis oligonucleotides GI-GATA, GII-GATA and GIII-GATA. Likewise, the GI- and GII-HS2-tk constructs were created from the HS2-tk. DR4-HS2-tk was constructed with the mutagenesis oligonucleotide VRE-DR4 from HS2-tk construct and from this, using the mutagenesis oligonucleotide GI-GATA, the GI-DR4-HS2-tk plasmid was generated. All constructs were checked using sequencing analysis.

\section{Acknowledgments}

We thank Joan Betz and members of the Stunnenberg lab for continued discussions and critical reading of the manuscript, and thank Stefan van den Akker for assistance. We also thank Aria Baniahmad for kindly providing us with L cells and the F2-tk construct, Sjaak Philipsen for MEL cells and Carl Figdor for K562 cells.
Chen JD and Evans RM. (1995). Nature, 377, 454- 457.

Chen HW, Smitmcbride Z, Lewis S, Sharif M and Privalsky ML. (1993). Mol. Cell. Biol., 13, 2366-2376.

Choi OR and Engel JD. (1988). Cell, 55, 17-26.

Ciana P, Braliou GG, Demay FG, von Lindern M, Barettino D, Beug H and Stunnenberg HG. (1998). EMBO J., 17, $7382-7394$.

Damm K, Thompson C and Evans RM. (1989). Nature, 339, $593-597$.

de Magistris L and Stunnenberg G. (1988). Nucleic Acids Res., 16, $3141-3156$.

Disela C, Glineur C, Bugge T, Sap J, Stengl G, Dodgson J, Stunnenberg H, Beug H and Zenke M. (1991). Genes Dev., 5, $2033-2047$.

Dolznig H, Bartunek P, Nasmyth K, Mullner EW and Beug H. (1995). Cell Growth Differ., 6, 1341-1352.

Emerson BM, Nickol JM, Jackson PD and Felsenfeld G. (1987). Proc. Natl. Acad. Sci. USA, 84, 4786-4790.

Fondell JD, Brunel F, Hisatake K and Roeder RG. (1996). Mol. Cell. Biol., 16, 281-287.

Fondell JD, Roy AL and Roeder RG. (1993). Genes Dev., 7, $1400-1410$.

Fuerstenberg S, Leitner I, Schroeder C, Schwarz H, Vennstrom B and Beug H. (1992). EMBO J., 11, $3355-$ 3365.

Gandrillon O, Ferrand N, Michaille JJ, Roze L, Zile MH and Samarut J. (1994). Oncogene, 9, 749-758.

Gandrillon O, Jurdic P, Pain B, Desbois C, Madjar JJ, Moscovici MG, Moscovici C and Samarut J. (1989). Cell, 58, $115-121$.

Gandrillon O, Schmidt U, Beug H and Samarut J. (1999). EMBO J., 18, 2764-2781. 
Geijtenbeek TB, Torensma R, van Vliet SJ, van Duijnhoven GC, Adema GJ, van Kooyk Y and Figdor CG. (2000). Cell, 100, $575-585$.

Glass CK and Rosenfeld MG. (2000). Genes Dev., 14, 121 141.

Goldhirsch A and Gelber RD. (1996). Semin. Oncol, 23, $494-505$

Heinzel T, Lavinsky RM, Mullen TM, Soderstrom M, Laherty CD, Torchia J, Yang WM, Brard G, Ngo SD, Davie JR, Seto E, Eisenman RN, Rose DW, Glass CK and Rosenfeld MG. (1997). Nature, 387, 43 - 48.

Horlein AJ, Naar AM, Heinzel T, Torchia J, Gloss B, Kurokawa R, Ryan A, Kamei Y, Soderstrom M, Glass CK and Rosenfeld MG. (1995). Nature, 377, 397-404.

Innis JW and Scott WA. (1983). Mol. Cell. Biol., 3, $2203-$ 2210.

Jenuwein T, Forrester WC, Fernandez-Herrero LA, Laible G, Dull M and Grosschedl R. (1997). Nature, 385, $269-$ 272.

Jeong S and Stein A. (1994). Nucleic Acids Res., 22, $370-$ 375.

Judelson C and Privalsky ML. (1996). J. Biol. Chem., 271, $10800-10805$.

Luckow B and Schutz G. (1987). Nucleic Acids Res., 15, 5490.

Nelson CC, Hendy SC, Faris JS and Romaniuk PJ. (1994). Mol. Endocrinol., 8, 829-840.

Nomenclature committee. (1999). Cell, 97, 161- 163.

Ogryzko VV, Schiltz RL, Russanova V, Howard BH and Nakatani Y. (1996). Cell, 87, $953-959$.

Olson DP, Sun B and Koenig RJ. (1998). J. Biol. Chem., 273, $3375-3380$.

Pain B, Melet F, Jurdic P and Samarut J. (1990). New Biol., 2, 284-294.

Philipsen S, Talbot D, Fraser P and Grosveld F. (1990). EMBO J., 9, 2159-2167.

Rascle A, Ghysdael J and Samarut J. (1994). Oncogene, 9, $2853-2867$.

Reeves R, Gorman CM and Howard B. (1985). Nucleic Acids Res., 13, 3599-3615.
Renaud JP, Harris JM, Downes M, Burke LJ and Muscat GE. (2000). Mol. Endocrinol., 14, 700-717.

Sap J, Munoz A, Schmitt J, Stunnenberg H and Vennstrom B. (1989). Nature, 340, $242-244$.

Schroeder C, Gibson L, Zenke M and Beug H. (1992). Oncogene, 7, 217-227.

Seraphin B and Kandels-Lewis S. (1996). Nucleic Acids Res., 24, 3276-3277.

Sharif M and Privalsky ML. (1991). Cell, 66, 885-893.

Stunnenberg HG, Garcia-Jimenez C and Betz JL. (1999). Biochim. Biophys. Acta., 1423, F15-F33.

Stunnenberg HG, Lange H, Philipson L, van Miltenburg RT and van der Vliet PC. (1988). Nucleic Acids Res., 16, $2431-2444$

Subauste JS and Koenig RJ. (1995). J. Biol. Chem., 270, $7957-7962$.

Subauste JS and Koenig RJ. (1998). Mol. Endocrinol., 12, $1380-1392$

Thormeyer D and Baniahmad A. (1999). Int. J. Mol. Med., 4 , $351-358$.

Urnov FD, Yee J, Sachs L, Collingwood TN, Bauer A, Beug H, Shi Y-B and Wolffe AP. (2000). EMBO J., 19, 40744090.

Van Lint C, Emiliani S, Ott M and Verdin E. (1996). EMBO $J ., 15,1112-1120$.

Wahlstrom GM, Harbers M and Vennstrom B. (1996). Oncogene, 13, 843-852.

Wahlstrom GM and Vennstrom B. (1998). Mol. Endocrinol., 12, 645-653.

Wang GF, Nikovits W, Jr, Schleinitz $\mathrm{M}$ and Stockdale FE. (1998). Mol. Cell. Biol., 18, 6023-6034.

Warrell RP, Jr, de The H, Wang ZY and Degos L. (1993). $N$. Engl. J. Med., 329, 177-189.

Wolff L. (1997). Biochim. Biophys. Acta., 1332, F67-F104.

Wong J, Patterton D, Imhof A, Guschin D, Shi Y and Wolffe A. (1998). EMBO J., 17, 520-534.

Zenke M, Munoz A, Sap J, Vennstrom B and Beug H. (1990). Cell, 61, 1035-1049.

Zhang J, Hu X and Lazar MA. (1999). Mol. Cell. Biol., 19, $6448-6457$ 